\title{
Elastic Interaction Models for Active Contours and Surfaces
}

\author{
Albert C.S. Chung ${ }^{1}$, Yang Xiang ${ }^{2}$, Jian $\mathrm{Ye}^{2}$, and W.K. Law ${ }^{1}$ \\ 1 Lo Kwee-Seong Medical Image Analysis Laboratory, \\ Department of Computer Science, \\ The Hong Kong University of Science and Technology, Hong Kong \\ 2 Department of Mathematics, \\ The Hong Kong University of Science and Technology, Hong Kong
}

\begin{abstract}
In this paper, we propose a new framework for active contour and surface models. Based on the concepts of the elastic interaction between line defects in solids, this framework defines an image-based speed field for contour evolution. Different from other level set based frameworks, the speed field is global and defined everywhere in the whole space. It can offer a long-range attractive interaction between object boundary and evolving contour. The new framework is general because it can be easily extended to higher dimension. Using the Fast Fourier Transforms, we also introduce an efficient algorithm for finding the values of the image-based speed field. Some experiments on synthetic and clinical images are shown to indicate the properties of our model.
\end{abstract}

\section{Introduction}

Extracting a surface from medical data is clinically an important step because the extracted boundary surface can provide essential ssvisual and quantitative information about the shape and size of the biological objects, e.g. brains and vessels.

Active contour models have widely been used for image segmentation and surface extraction. The classical snakes approach evolves and finds object boundary based on forces derived from the internal energy of the contour and local image gradient [4].

If the moving contour is not close enough to the target object, the capture range of the contour can be limited. Then, an additional constant balloon force was introduced along the contour normal direction to accelerate the contour motion and increase the capture range [1].

To offer more flexibility in handling multiple objects and their topological changes, the level set framework [6.5] was used to model the moving contour. The dynamic equation is given as

$$
\frac{\partial \phi}{\partial t}=g(\nabla I)\left(\nabla \cdot\left(\frac{\nabla \phi}{|\nabla \phi|}\right)+\nu\right)|\nabla \phi|,
$$

where $\phi(x, y)$ is the level set function whose zero level set represents the contour. All terms before $|\nabla \phi|$ gives the speed of the embedded contour along its normal 
direction. The first term $\nabla \cdot\left(\frac{\nabla \phi}{|\nabla \phi|}\right)$ smooths and shortens the contour based on contour curvature. The second term $\nu$ is the constant balloon force making the contour expand or shrink depending on its sign. The gradient function $g(\nabla I)$ gives very small value at the boundary and makes the speed of the moving contour small. As such, the evolving contour will stop on the object boundary. However, without the constant balloon force, the capture range can be short and the contour cannot reach the narrow concave regions of the object boundary. This is because the effect of $\nabla I$, defined by $g(\nabla I)$ in Eq. 1, is localized near the boundary. While with the balloon force, there is a limitation that the balloon force cannot make some parts of contour shrink while other parts of the contour expand. Therefore, the initial contour must be placed entirely outside or inside the object to be detected.

In this paper, inspired by the elastic interaction between line defects in solids, we propose an active contour method offering a long-range attractive interaction between two contours (object boundary and evolving contour). We shall define a long-range attraction generated by the object boundary and acting on the evolving contour for solving the segmentation problem. The speed field due to this interaction is calculated efficiently using the Fast Fourier Transforms, and is defined everywhere in the space. No force extension is needed, as it is commonly needed in the conventional level set based active contour methods. We also use the level set framework to handle the topological changes during the contour evolution. This framework is general and can be applied to the Ndimensional segmentation problems. It is experimentally shown that the method can be effective in detecting elongated and tubular structures, e.g. brain vessels.

\section{Method}

\subsection{D Segmentation Based on Elastic Interaction}

The main goal of this section is to define a speed field $v$ for encouraging attractive interaction between object boundary and moving contour during the contour evolution. The level set framework is employed to represent the moving contours in $2 \mathrm{D}$ and surfaces in 3D [6]. Let $\phi$ be the level set function. The evolution equation is given as $\frac{\partial \phi}{\partial t}=v|\nabla \phi|$, where $v$ represents a speed field, in which the values of speed are well defined in the whole space.

We now define the speed field $v$ of a two dimensional moving contour. As shown in Fig. 1, the (blue) contour $\gamma(s)$ represents the object boundary and another (red) contour represents the moving contour. At any point $\mathbf{P}$ on the moving contour, the speed is derived based on the elastic interaction between line defects in solids [2] and our previous work [10]. The speed field $v$ is defined as

$$
v=-\int_{\gamma(s)} \frac{\mathbf{r} \cdot \mathbf{n}}{r^{3}} d s,
$$

where $\mathbf{r}$ is a vector between point $\mathbf{P}$ and a point on $\gamma(s), r=|\mathbf{r}|$ denotes the Euclidean distance between these two points, and $\mathbf{n}$ represents the normal di- 


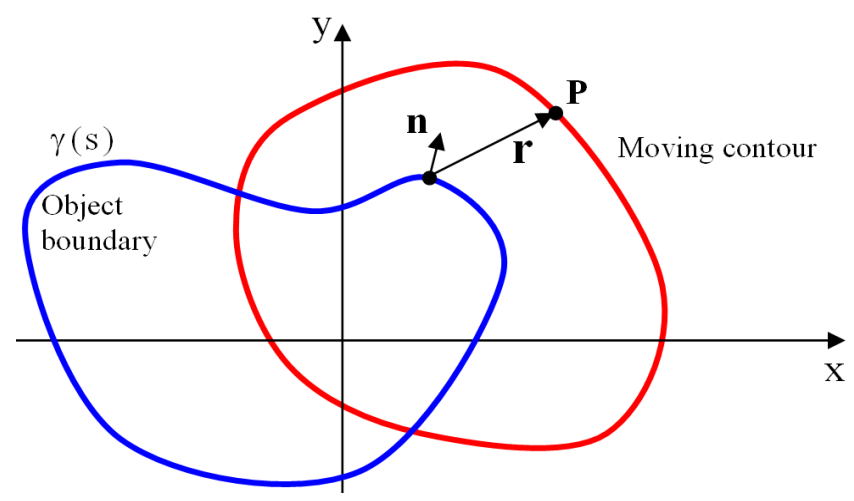

Fig. 1. Elastic interaction between object boundary (blue) and moving contour (red)

rection. Under this definition, the speed inside the object boundary and outside the object boundary are different in sign.

We describe how the speed function defined above can be used for image segmentation problem. Let an image be $I(x, y)$ located in the $z=0$ plane, where $(x, y) \in \Omega$ and $\Omega$ denotes the image domain. The speed field is set to depend on the intensity values in the image by replacing the normal direction $\mathbf{n}$ in Eq. 2 by the image gradient $\nabla I$. However, the image-based speed function is singular on the contour $\gamma(s)$. The singularities can then be smeared out if the normal direction $\mathbf{n}$ is replaced with the gradient of the smoothed image $\nabla\left(G_{\sigma} * I\right)$, where $G_{\sigma}$ represents a Gaussian smoothing filter with standard deviation $\sigma$. Therefore, the image-based speed field $v$ is given as

$$
v=-\int_{\Omega} \frac{\mathbf{r} \cdot \nabla\left(G_{\sigma} * I\right)}{r^{3}} d x d y
$$

where $\Omega$ denotes the image domain and $(x, y) \in \Omega$.

Another important property of the speed field $v$ is that it is a long range speed field generated by the object boundary, and there is no need to place the initial contour entirely inside or outside the object. Thus the moving contour can "see" the far away boundary by the interaction between object boundary and evolving contour boundary. Also, the sign of the speed depends on the direction of the contour and the object boundary, so that the contour is not necessarily to be placed entirely inside or near the object boundary. The direction of the object boundary $\mathbf{t}$ is defined as

$$
\mathbf{t}=\frac{1}{\left|\nabla\left(G_{\sigma} * I\right)\right|}\left(\frac{\partial\left(G_{\sigma} * I\right)}{\partial y},-\frac{\partial\left(G_{\sigma} * I\right)}{\partial x}, 0\right) .
$$

For instance, if an object has a stronger intensity than the background, the direction of the object boundary is counterclockwise; and is clockwise vice versa. The direction of the moving contour, i.e. the zero level contour of the level set 
function $\phi(x, y)$, can also be defined similarly, with $G_{\sigma} * I$ replaced by $\phi$ in Eq. 4. The level set function is chosen such that the moving contour has an opposite direction with respect to the object boundary. As a result, the moving contour is attracted to the object boundary under the speed field $v$.

In the above definition of speed function, the image noise also generates a speed field for the moving curve, resulting in spurious contours. The speed generated by the noise is relatively small as compared with that by the object boundary. We remove this contribution of the noise by adding the interaction within the moving contour, so that the relative weak interaction between the noise and the moving contour can be overcome. The speed field $v$ is now defined as

$$
v=-\int_{\Omega} \frac{\mathbf{r} \cdot \nabla\left(G_{\sigma} * I+w H(\phi)\right)}{r^{3}} d x d y,
$$

where $w$ is an adjustable coefficient and $H$ is the Heaviside function, which is defined as

$$
H(\phi)= \begin{cases}0 & \text { if } \phi \leq-\epsilon, \\ \frac{1}{2}\left(\sin \left(\frac{\pi \phi}{2 \epsilon}\right)+1\right) & \text { if }-\epsilon<\phi<\epsilon, \\ 1 & \text { if } \phi \geq \epsilon,\end{cases}
$$

where $\epsilon$ is a small constant.

The values of the speed field $v$ can be solved efficiently using the Fast Fourier Transform (FFT) algorithm. It is well known that in three dimensional space, $-1 / 4 \pi r$ is the solution of the Poisson equation,

$$
-\triangle \frac{1}{4 \pi r}=\delta
$$

where $\delta$ is the three dimensional Dirac delta function. Performing the Fourier transformations on both sides of the above equation, we can get

$$
\frac{\widehat{1}}{r}=\frac{1}{m^{2}+n^{2}+l^{2}} \cdot \frac{1}{2 \pi^{2}},
$$

where $m, n$, and $l$ are frequencies in the Fourier space. Thus

$$
\widehat{\nabla \frac{1}{r}}=\frac{(i m, i n, i l)}{m^{2}+n^{2}+l^{2}} \cdot \frac{1}{2 \pi^{2}} .
$$

In order to perform the FFT algorithm to get the values of speed (Eq. 5), we have to change our formulation from two dimensional space to three dimensional space. To achieve this goal, we multiply a factor $\delta(z)$, where $\delta(z)$ is one-dimensional Dirac delta-function. Therefore, Eq. 5 can be re-written as

$$
v=-\int_{z} \int_{\Omega} \frac{\mathbf{r}}{r^{3}} \cdot\left(\nabla\left(G_{\sigma} * I(x, y)+w H(\phi)(x, y)\right), 0\right) \delta(z) d x d y d z .
$$

Let the Fourier coefficients of the function $G_{\sigma} * I+w H(\phi)$ be $\left\{d_{m n}\right\}$. The Fourier coefficients of the function $\left(\nabla\left(G_{\sigma} * I(x, y)+w H(\phi)(x, y)\right), 0\right)$ are then 
$\left\{\left(i m d_{m n}, i n d_{m n}, 0\right)\right\}$. Performing the Fourier transformation on both sides of Eq. [10, based on Eq. 9], we get

$$
\begin{aligned}
\widehat{v} & =\frac{(i m, i n, i l)}{m^{2}+n^{2}+l^{2}} \cdot \frac{1}{2 \pi^{2}} \cdot\left(i m d_{m n}, i n d_{m n}, 0\right) \cdot \frac{1}{2 \pi} \cdot(2 \pi)^{2} \\
& =-\frac{1}{\pi} \cdot \frac{m^{2}+n^{2}}{m^{2}+n^{2}+l^{2}} d_{m n} .
\end{aligned}
$$

Finally, we have

$$
\begin{aligned}
v(x, y) & =-\int_{-\infty}^{\infty} \sum_{m, n} \frac{1}{\pi} \frac{m^{2}+n^{2}}{m^{2}+n^{2}+l^{2}} d_{m n} e^{i m x} e^{i n y} d l \\
& =-\sum_{m, n} \frac{\sqrt{m^{2}+n^{2}}}{2} \cdot d_{m n} e^{i m x} e^{i n y}
\end{aligned}
$$

To obtain a smooth moving contour, we can introduce a small curvature term associated with a small weight $\mu$. Now, the evolution equation is given as

$$
\frac{\partial \phi}{\partial t}=\left(\mu \nabla \cdot\left(\frac{\nabla \phi}{|\nabla \phi|}\right)+v\right)|\nabla \phi|
$$

\subsection{Extension to 3D Segmentation Problem}

Similarly, we define our three dimensional speed field $v$ as

$$
v=-\int_{S} \frac{\mathbf{r} \cdot \mathbf{n}}{r^{4}} d A
$$

where $S$ represents the object boundary in 3D. By replacing the normal direction $\mathbf{n}$ by the gradient of the smoothed image $\nabla\left(G_{\sigma} * I\right)$, the value of speed $v$ is now relying on the image intensity values in an image volume $I(x, y, z)$, where $(x, y, z) \in \Omega$ and $\Omega$ denotes the image domain. In order to perform FFT algorithm to solve the image-based speed function, we have to change the formulation to $4 \mathrm{D}$ space. The speed function is re-written as

$$
v=-\int_{w} \int_{\Omega} \frac{\mathbf{r}}{r^{4}} \cdot\left(\nabla\left(G_{\sigma} * I(x, y, z)+w H(\phi)(x, y, z)\right), 0\right) \delta(w) d x d y d z d w
$$

where $\delta(w)$ is the one-dimensional Dirac delta-function. Let the Fourier coefficients of the function $G_{\sigma} * I+w H(\phi)$ be $\left\{d_{m n l}\right\}$. Similarly to $2 \mathrm{D}$ problem described in the previous section, performing FFT on both sides of Eq. 16] and following by taking the inverse FFT, we get

$$
v(x, y, z)=-\sum_{m, n, l} \frac{\sqrt{m^{2}+n^{2}+l^{2}}}{8} \cdot d_{m n l} e^{i m x} e^{i n y} e^{i l z},
$$

where $m, n$, and $l$ are frequencies in the Fourier space. 


\subsection{Numerical Implementation}

For the numerical implementation of Eq.(14), we use central difference for the curvature term, Godunov's scheme 7 combined with fifth order WENO derivative [3] for the term $v|\nabla \phi|$, and the forward Euler method in time. Re-initialization for $\phi$ is used to reduce the numerical errors [9. The initial zero level contour of $\phi$ is set to be the zero crossing of the speed field generated by the image only, i.e. $\phi=v /|v|$, where $v$ is calculated using $w=0$. This gives an initial contour very close to the object boundary, so that the object boundary can then be found and the noise be removed efficiently after short-time evolution.

\section{Results}

The proposed method has been applied to synthetic images and four sets of 3D Rotational Angiographic (RA) images. We have also implemented the gradient vector flow (GVF) method [12118]. Since the results using these GVF methods [12[118] are similar in the comparisons, all results are obtained using the modified GVF method with the balloon force 8 .

\subsection{Synthetic Images}

In this section, all results were obtained using a numerical mesh with the size of $128 \times 128$ pixels. The width and length were equal to 2 units. The pixel width was $d x=d y=2 / 128$.

Fig. 2 shows the results on an image with multiple objects, which have different intensity and topology, but without noise. Given that the intensity values range between 0 (white) and 1 (black), the intensity values of the four objects were $3 / 9,4 / 9,5 / 9$ and $6 / 9$ (see Fig. 2 (a)). As shown in the figure, the image has thin features such as convex (bottom left) and concave (top right) regions. The parameters of our method were set as follows: $\sigma=0.8$ (Eq. [5), $w=0.35$ (Eq. (5)), $\epsilon=d x$ (Eq. (6) and $\mu=0.002$ (Eq. 14). Our method found the object boundaries accurately (see Fig. 2(b)). In fact, for this image without noise, the zero level contour of the speed field $v$ gives the object boundary directly. In this case, no evolution is needed. Fig. 2(c) shows that the speed field is globally defined and can have long influence on the moving contour.

The bottom row of Fig. 2 2 shows the results obtained using the GVF method with adaptive bidirectional balloon force. Unlike our method, this method needs extension of force (velocity) field based on the diffusion of gradient information. The parameters were set as follows: $\mu=0.02, \sigma_{E}=2 d x, \beta=0.002$ and $\epsilon=0.1$ (see [8 for definitions of the parameters). We set a circle as the initial contour (see Fig. 2(d)). As shown in Fig. 2(e), not all parts of the contour were attracted to the correct boundaries of the objects (see the bottom left object in Fig. 2(e)). Fig. 2(f) shows the corresponding force field of the GVF method. From the figure, it is observed that, at the middle between two adjacent objects, the contour cannot move further. This is because the extended velocity is perpendicular to the normal direction of the zero level set. 


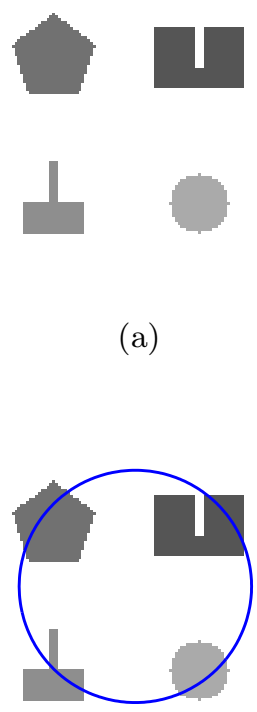

(d)

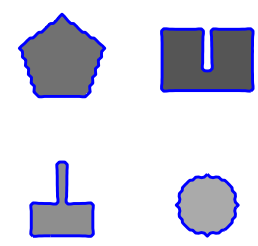

(b)

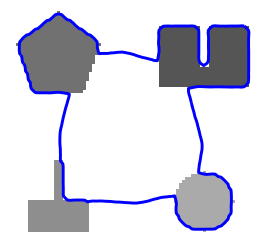

(e)

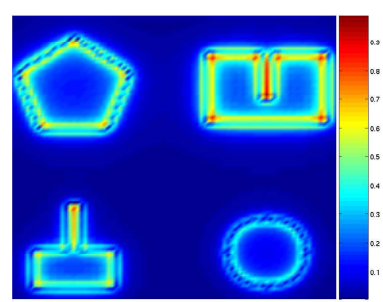

(c)

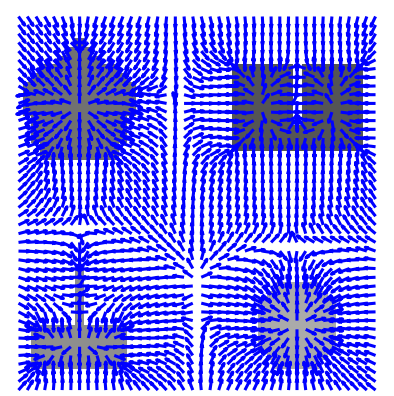

(f)

Fig. 2. (a) An input image with multiple objects, (b) results obtained using our method, (c) speed field $v$ of our method (Note that $\log _{2}(|v|+1$ ) is shown for better visualization.), (d) initial contour for GVF method, (e) results obtained using the GVF method and (f) GVF force field

Our method has been applied to the same image (see Fig. 2(a)) but with different levels of noise. The SD of the background noise $\sigma_{B}=0.0621$ was obtained from the real 3D-RA images, which will be described in the next section.

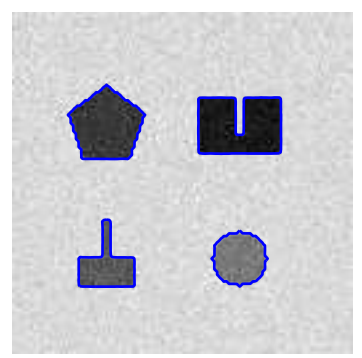

(a)

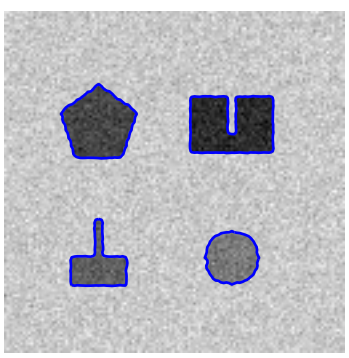

(b)

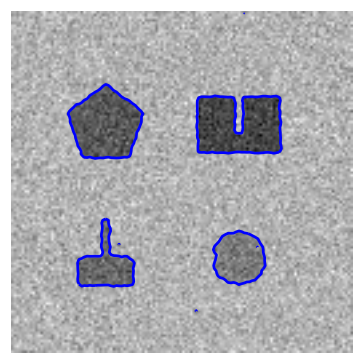

(c)

Fig. 3. Synthetic images with different levels of noise: (a) $0.5 \sigma_{B}$, (b) $\sigma_{B}$ and (c) $2 \sigma_{B}$. Intensity values range between 0 (white) and 1 (black) in the images. 


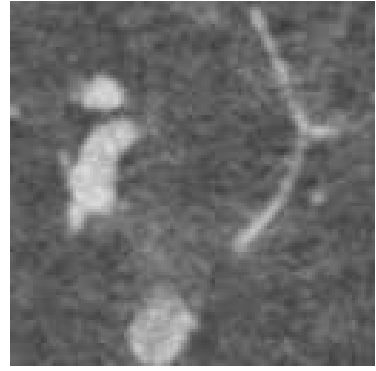

(a)

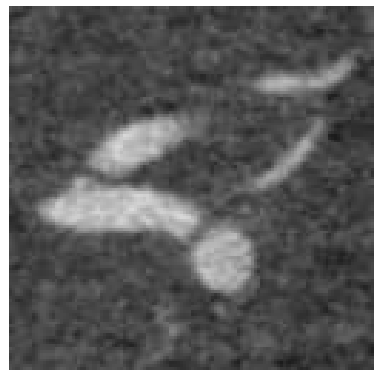

(c)

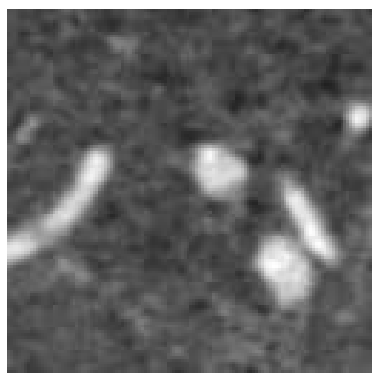

(g)

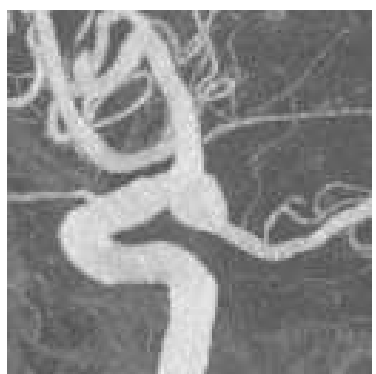

(e)

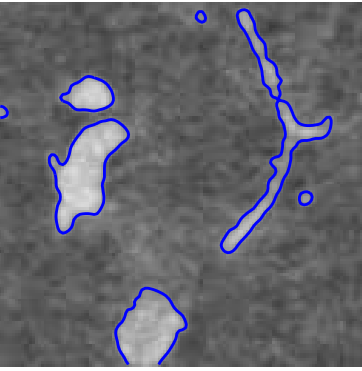

(b)

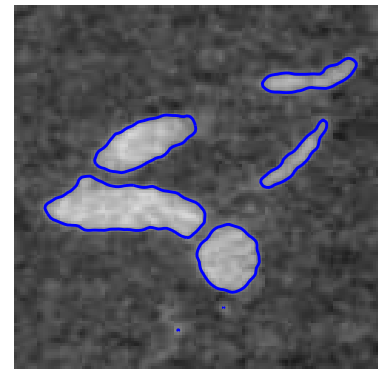

(d)

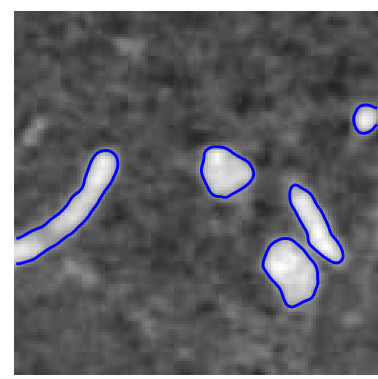

(h)

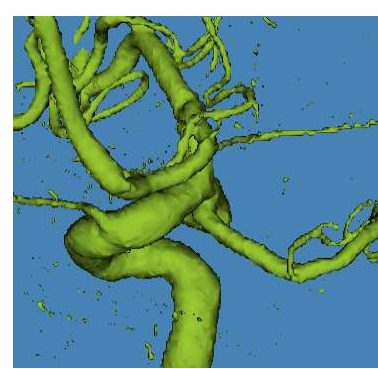

(f)

Fig. 4. Image slices from 3DRA image volumes. Original slices for patients 1 (Fig.a), 2 (Fig.c) and 3 (Fig.e). Results obtained using our method for patients 1 (Fig.b), 2 (Fig.d) and 3 (Fig.f). Fig.g and Fig.h show the MIP image and the corresponding 3D view of the result obtained using our method. 


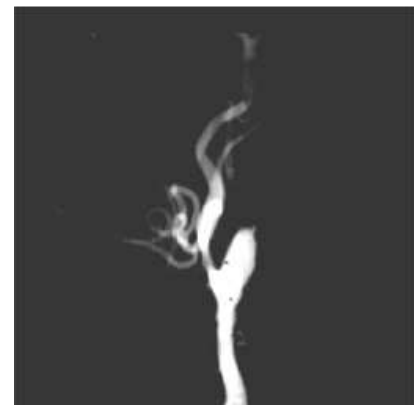

(a)

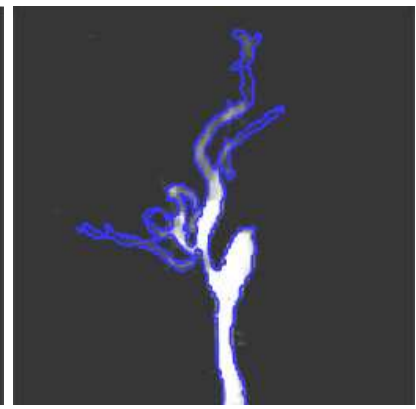

(b)

Fig. 5. (Fig.a) DSA vascular image. (Fig.b) The corresponding segmentation result.

The errors of segmentation were $0 \%, 0.06 \%$ and $0.63 \%$ for Figs. [3(a), 3)(b) and 3(c) respectively. It shows that the proposed method can give reasonable segmentation results when noise level increases.

\subsection{Real Images}

We have applied our 3D segmentation method on four 3D-RA clinical datasets acquired by a Philips Integris Imager medical system at the Department of Diagnostic Radiology and Organ Imaging, Prince of Wales Hospital, Hong Kong. The data volume was around $100 \times 100 \times 100$ voxels with a voxel size of $0.75 \times 0.75 \times$ $0.75 \mathrm{~mm}^{3}$. Figs. 4(a), 4(c) and 4(e) show three selected image slices. Contours obtained using our method are shown in Figs. 4(b), 4(d) and 4(f) respectively (note that the contours are lying inside the vascular regions). The initial contours were obtained directly using the speed field, and then evolved using the level set method and FFT for speed field calculation. Given that the vasculature has convex and concave structures, the results of segmentation are satisfactory. Fig. [5] shows a DSA vascular image with thin and elongated structures, and the intensity values are low (weak linkage) in the upper half of the image. As shown in the figure (Fig. 50 b), our method can help capture weak edges connected along the strong edges. A 3D surface of one of the segmented image volumes and the corresponding MIP are shown in Figs. 4(g) and 4(h).

\section{Conclusion}

We have proposed the use of elastic interaction for active contour and surface models. Our method has been applied to the synthetic and real image volumes for image segmentation. The experimental results show that, for images without noise (e.g. synthetic images), the zero level set of the speed function can give contour very near to the target object boundary. For images with noise (e.g. real images), the initial contour can be efficiently computed and then object 
details can be detected via contour evolution using the Fast Fourier Transform (FFT) algorithm. It is experimentally shown that our method can be effective in detecting elongated and tubular structures, e.g. brain vessels in 3DRA or DSA.

\section{References}

1. L.D. Cohen. On active contour models and balloons. Computer Vision and Graphic Image Processiong Conference, 53(2):211-331, 1991.

2. J.P. Hirth and J. Lothe. Theory of Dislocations, 2nd edition. John Wiley \& Sons, Inc., New York, 1982.

3. G.S. Jiang and D. Peng. Weighted ENO schemes for Hamilton-Jacobi equations. SIAM Journal on Scientific Computing, 21(6):2126-2143, 2000.

4. M. Kass, A. Witkin, and D. Terzopoulos. Snakes: Active contour models. International Journal of Computer Vision, 1(4):321-331, 1988.

5. R. Malladi, J.A. Sethian, and B.C. Vemuri. Shape modeling with front propagation: A level set approach. IEEE Transactions on Pattern Analysis and Machine Intelligence, 17(2):158-175, 1995.

6. S. Osher and J.A. Sethian. Fronts propagating with curvature-dependent speed: algorithms based on Hamilton-Jacobi formulations. Journal of Computational Physics, 79:12-49, 88.

7. S. Osher and C.W. Shu. High-order essentially nonoscillatory schemes for Hamilton-Jacobi equations. SIAM Journal of Numerical Analysis, 28(4):907-922, 1991.

8. N. Paragios, O. Mellina-Gottardo, and V. Ramesh. Gradient vector flow fast geometric active contours. IEEE Transactions on Pattern Analysis and Machine Intelligence, 26(3):402-407, 2004.

9. D. Peng, B. Merriman, S. Osher, H.K. Zhao, and M. Kang. A PDE-based fast local level set method. Journal of Computational Physics, 155(2):410-438, 1999.

10. Y. Xiang, A.C.S. Chung, and J. Ye. A New Active Contour Method based on Elastic Interaction. IEEE International Conference on Computer Vision and Pattern Recognition, 1:452-457, 2005.

11. C. Xu and J.L. Prince. Generalized gradient vector flow external forces for active contours. Signal Processing, 71(2):131-139, 1998.

12. C. Xu and J.L. Prince. Snakes, shapes, and gradient vector flow. IEEE Transaction on Image Processing, 7(3):359-369, 1998. 